\title{
Cardioprotection via adaptation to hypoxia: expanding the timeline and targets?
}

\author{
Karin Przyklenk • Peter Whittaker
}

Received: 17 February 2011/ Accepted: 18 February 2011/Published online: 10 March 2011

(C) Springer-Verlag 2011

Substantial effort and resources throughout the past four decades have been devoted to the investigation of novel paradigms to limit the damage caused to the heart by myocardial ischemia and, more recently, the spectrum of lethal ischemia-reperfusion injury. The scope of this intellectual and financial investment is underscored by the fact that, as of December 2010, more than 9,600 publications cited in PubMed include the term 'cardioprotection' as a key word.

A milestone in the field of cardioprotection was provided in 1986 by Murry et al. [14], who demonstrated that transient exposure of the heart to a stressful stimulus (i.e., antecedent 'preconditioning' ischemia) marshaled a profound, endogenous cardioprotective response and rendered the myocardium resistant to a subsequent sustained ischemic insult. Although this observation was, at the time, considered remarkable and viewed with some skepticism, the phenomenon of cardioprotection via adaptation to stress has been corroborated in a host of studies and expanded from the initial paradigm of ischemic preconditioning to encompass multiple triggers including remote ischemia or 'preconditioning at a distance' $[18,27]$, mild hypothermia and hyperthermia [12, 23, 25, 29], endotoxin

K. Przyklenk $(\bowtie) \cdot$ P. Whittaker

Cardiovascular Research Institute, Wayne State University

School of Medicine, Elliman Building, Room 1107,

421 E Canfield, Detroit, MI 48201, USA

e-mail:kprzykle@med.wayne.edu

\section{K. Przyklenk}

Department of Physiology, Wayne State University School of Medicine, Detroit, MI, USA

K. Przyklenk · P. Whittaker

Department of Emergency Medicine, Wayne State University

School of Medicine, Detroit, MI, USA and endotoxin analogs $[19,30,31]$, endoplasmic reticulum stress [17] and many others. There are, however, two important caveats to the reduction of infarct size achieved with ischemic preconditioning and other stressors: the protective triggers are considered to delay (rather than preclude) cardiomyocyte death, and efficacy is achieved when the triggers are applied as a pretreatment (before ischemia) or, in some instances, during the initial secondsminutes of reperfusion. That is: myocardial salvage is typically not obtained in the setting of permanent coronary occlusion, and cardioprotective strategies are generally ineffective when administered hours-days following relief of ischemia.

In the current issue of Basic Research in Cardiology, Xu and colleagues [28] report that prolonged exposure of rats to intermittent hypobaric hypoxia (IHH: barometric pressure of $404 \mathrm{mmHg}$ and $\mathrm{PO}_{2}$ of $84 \mathrm{mmHg}$ [corresponding to an altitude of $5,000 \mathrm{~m}], 6 \mathrm{~h} /$ day, for 14 or 28 days) was associated with a significant reduction in myocardial infarct size and improvement in contractile performance. The concept that intermittent hypoxia, either alone or in combination with reduced atmospheric pressure, can initiate a cardioprotective response is not novel: infarct size reduction in response to hypoxia is well-documented in the literature $[7,9,10,15,16]$. However, the study by Xu et al. [28] is noteworthy, in that the authors used a model of permanent coronary artery ligation rather than ischemiareperfusion, and daily exposure to IHH was begun at 7 days after the onset of ischemia.

The obvious question is: how can this intriguing observation be explained? Xu and coworkers speculate that the observed evidence of angiogenesis (reflected by increases in vascular endothelial growth factor (VEGF) expression and capillary density in the peri-infarct region), manifest at 14 and 28 days after the onset of treatment, 
may have played a role. In addition, the authors provide concomitant evidence of increased phosphorylation of heat shock protein 27 and reduction of apoptotic cell death as detected by TUNEL staining at the infarct margins [28]. When these data are considered within the framework of the well-defined, rapid temporal progression of ischemiainduced cardiomyocyte death in the rat heart (i.e., development of a transmural infarct within $\sim 2-4 \mathrm{~h}$ after the onset of coronary occlusion [4, 23]), direct salvage of ischemic myocardium by IHH-induced increases in myocardial perfusion or up-regulation of cardioprotective signaling appears improbable. There are, however, at least three alternative explanations that warrant consideration.

The first and fundamental issue is the requisite documentation that the primary determinants of infarct size are comparable among all study groups. In the rat, as in other in vivo models, this includes the measurement of risk region, or volume of myocardium rendered ischemic at the time of coronary artery occlusion. Accurate quantitation of risk region in long-term studies is notoriously challenging: in many instances (including the current study), the assumption is made that the lateral extent of the infarct scar assessed days-weeks post-occlusion coincides with the margins of the risk region. Unfortunately this assumption can be problematic. For example: we measured the lateral distance between the margin of the infarct (assessed with triphenyl tetrazolium staining) and the edge of the risk region (assessed with blue pigment infusion) in cohorts of control and preconditioned rats subjected to $90 \mathrm{~min}$ of sustained ischemia followed by $4.1 \mathrm{~h}$ of reperfusion [27]. In control hearts, this distance was independent of the size of the risk region and was approximately $0.4 \mathrm{~mm}$ on each side of the infarct. In hearts that underwent ischemic preconditioning, the lateral distance was inversely proportional to risk region (the smaller the risk region the larger the distance) and, for risk regions in the range of 20-25\% of the left ventricle, was approximately $2.5 \mathrm{~mm}$ on each side [27]. Accordingly, the assumption that the lateral edges of the infarct (or scar) abut the margins of the ischemic territory may provide an underestimate of the risk region. This assumption could be further compromised by an intervention-particularly a therapy such as IHH that stimulates angiogenesis and alters coronary blood flow in the peri-infarct region-and thus could confound interpretation of the results.

The improved myocardial viability, reduction in scar area and attenuated left ventricular dysfunction reported by $\mathrm{Xu}$ et al. with IHH are reminiscent of results obtained with cell-based therapies including activation of endogenous cardiac progenitor cells, administration of exogenous progenitor cells, or recruitment and homing of bone marrow progenitor cells to the infarcted heart $[8,21]$. In addition, a substantial body of evidence suggests that 'hypoxic preconditioning' of progenitor cells (specifically, exposure of these cells to 24-72 $\mathrm{h}$ of hypoxia before administration), renders the cells more tolerant to the unfavorable conditions of the ischemic myocardium and augments the regenerative benefits of cell therapy [2, 22]. These data raise the interesting possibility that, while direct salvage of ischemic myocytes is unlikely, the favorable effects of IHH in this paradigm of very late treatment may be due in part to a hypoxia-induced activation or mobilization of resident populations of progenitor cells. This concept is, however, speculative and will require future prospective investigation.

A third possibility is that IHH directly affected the scar. For example, there is evidence that cultured murine fibroblasts exposed to hypoxia can change their phenotype to myofibroblasts [13]. Although such cells produce collagen, it is the contractile ability of $\alpha$-smooth muscle actin which may be of particular relevance as myofibroblasts are known to produce scar contracture [5]. It is well-recognized that myocardial scars can undergo considerable shrinkage under normal circumstances. For instance, in pigs, a 30-60\% reduction in tissue volume within the infarct was reported at 3 weeks after infarction, with the principal direction of shrinkage being circumferential [6]. If such scar shrinkage was enhanced through $\mathrm{IHH}$-associated changes in the myofibroblast population, the net result, in terms of the appearance of a ventricular cross-section slice, would be the same as reduction of infarct size. Identification of an effect of IHH on the scar, rather than on cardiomyocyte salvage or recruitment of progenitor cells, might be achieved by the measurement of the circumferential extent of non-infarcted muscle in a ventricular cross-section. This point is illustrated by a study from our group in which externally-mediated scar shrinkage using the thermal effects of a laser significantly reduced circumferential scar length when compared with untreated hearts, yet circumferential length of non-infarcted muscle did not differdata that are consistent with equal infarct size in both groups prior to treatment [26].

If IHH augments scar shrinkage, this raises a closely related concept: scar contracture requires extensive remodeling of the collagen matrix, including both collagen production and collagen degradation. Hypoxia has been shown to stimulate collagen synthesis by cultured human vascular-derived myofibroblasts [24]. In contrast, the effects on matrix metalloproteinases have been mixed: several studies reported increases in MMP-2 synthesis in cultured rodent fibroblasts [1, 13], whereas exposure of cultured human myofibroblasts to chronic hypoxia attenuated MMP-2 activation [20]. The overall effect of hypoxia on collagen deposition is, however, best assessed in the intact heart. In this regard, non-ischemic hearts of rats exposed to IHH (18 h/day for 10 days to a barometric 
pressure of $0.4 \mathrm{~atm}(308 \mathrm{mmHg})$ and $\mathrm{PO}_{2}$ of $64.5 \mathrm{mmHg}$ [equivalent to 7,000 $\mathrm{m}$ altitude]) displayed an increase in myocardial collagen content as reflected by a doubling in the hydroxyproline content of the left ventricle [3].

Interestingly, in the study by $\mathrm{Xu}$ et al. [28], collagen content expressed as a percent of the total left ventricle (including both scar and remote non-infarcted myocardium) was reduced, rather than increased, with IHH treatment. The obvious difference between the two protocols that may contribute to the seemingly discrepant outcomes is the assessment of collagen content in normally perfused hearts [3] versus hearts subjected to permanent coronary artery ligation [28]. This may imply that IHH has a differential, biphasic effect on collagen deposition in noninfarcted normally perfused regions of the heart versus scar, possibly due to the superimposition of an intermittent hypoxic stimulus on a region of the heart rendered chronically hypoxic by coronary artery occlusion. Alternatively, IHH may have selectively increased the amount of perivascular collagen in intramyocardial arteries [11], an effect that could favorably limit infarct expansion in the absence of an overall increase in scar collagen. Evaluation of these concepts would require discrete regional quantitation of collagen content in the scar, in non-infarcted myocardium, and around blood vessels-analyses that were not incorporated into the current study.

The seminal reports that brief preconditioning ischemia, applied locally or remotely, could protect the heart against infarction were subjected to intense scrutiny. However, these observations, once verified, yielded a paradigm shift in the field of cardioproptection and in our understanding of adaptation of the heart to stressors. The finding by $\mathrm{Xu}$ and colleagues that IHH (or, indeed, any therapeutic strategy), applied at 7 days after permanent coronary artery ligation, can mitigate the deleterious consequences of sustained myocardial ischemia is equally remarkable. However, if substantiated and if mechanistic insights are achieved, these data raise the intriguing possibility that a relatively simple intervention-intermittent exposure to simulated altitude, initiated days after coronary artery occlusion-may offer profound benefit to patients with acute myocardial infarction.

\section{References}

1. Bergman MR, Cheng S, Honbo N, Piacentini L, Karliner JS, Lovett DH (2003) A functional activating protein 1 (AP-1) site regulates matrix metalloproteinase 2 (MMP-2) transcription by cardiac cells through interactions with JunB-Fra1 and JunB-FosB heterodimers. Biochem J 369:485-496. doi:10.1042/BJ20020707

2. Chacko SM, Ahmed S, Selvendiran K, Kuppusamy ML, Khan M, Kuppusamy P (2010) Hypoxic preconditioning induces the expression of prosurvival and proangiogenic markers in mesenchymal stem cells. Am J Physiol Cell Physiol 299:C1562-C1570. doi:10.1152/ajpcell.00221.2010

3. Genovese A, Latte S, Bozzaotre M, Chiariello M (1983) Response of the left ventricular connective tissue to hypoxia. Res Exp Med (Berl) 183:111-115

4. Hearse DJ, Richard V, Yellon DM, Kingma JG Jr (1988) Evolving myocardial infarction in the rat in vivo: an inappropriate model for the investigation of drug-induced infarct size limitation during sustained regional ischaemia. J Cardiovasc Pharmacol 11:701-710

5. Hinz B (2007) Formation and function of the myofibroblast during tissue repair. J Invest Dermatol 127:526-537. doi: 10.1038/sj.jid.5700613

6. Holmes JW, Yamashita H, Waldman LK, Covell JW (1994) Scar remodeling and transmural deformation after infarction in the pig. Circulation 90:411-420

7. Kolar F, Jezkova J, Balkova P, Breh J, Neckar J, Novak F, Novakova $\mathrm{O}$, Tomasova $\mathrm{H}$, Srbova $\mathrm{M}$, Ost'adal B, Wilhelm J, Herget J (2007) Role of oxidative stress in PKC-delta upregulation and cardioprotection induced by chronic intermittent hypoxia. Am J Physiol Heart Circ Physiol 292:H224-H230. doi: 10.1152/ajpheart.00689.2006

8. Leri A, Hosoda T, Rota M, Kajstura J, Anversa P (2007) Myocardial regeneration by exogenous and endogenous progenitor cells. Drug Discov Today Dis Mech 4:197-203. doi: 10.1016/j.ddmec.2008.02.008

9. Mallet RT, Ryou MG, Williams AG Jr, Howard L, Downey HF (2006) Beta1-Adrenergic receptor antagonism abrogates cardioprotective effects of intermittent hypoxia. Basic Res Cardiol 101:436-446. doi:10.1007/s00395-006-0599-y

10. Manukhina EB, Downey HF, Mallet RT (2006) Role of nitric oxide in cardiovascular adaptation to intermittent hypoxia. Exp Biol Med (Maywood) 231:343-365 pii: 231/4/343

11. Matsumoto C, Hayashi T, Kitada K, Yamashita C, Miyamura M, Mori T, Ukimura A, Ohkita M, Jin D, Takai S, Miyazaki M, Okada Y, Kitaura Y, Matsumura Y (2009) Chymase plays an important role in left ventricular remodeling induced by intermittent hypoxia in mice. Hypertension 54:164-171. doi: 10.1161/HYPERTENSIONAHA.109.131391

12. Miki T, Liu GS, Cohen MV, Downey JM (1998) Mild hypothermia reduces infarct size in the beating rabbit heart: a practical intervention for acute myocardial infarction? Basic Res Cardiol 93:372-383

13. Misra S, Fu AA, Misra KD, Shergill UM, Leof EB, Mukhopadhyay D (2010) Hypoxia-induced phenotypic switch of fibroblasts to myofibroblasts through a matrix metalloproteinase $2 /$ tissue inhibitor of metalloproteinase-mediated pathway: implications for venous neointimal hyperplasia in hemodialysis access. J Vasc Interv Radiol 21:896-902. doi:10.1016/j.jvir.2010.02.030

14. Murry CE, Jennings RB, Reimer KA (1986) Preconditioning with ischemia: a delay of lethal cell injury in ischemic myocardium. Circulation 74:1124-1136

15. Neckar J, Markova I, Novak F, Novakova O, Szarszoi O, Ost'adal B, Kolar F (2005) Increased expression and altered subcellular distribution of PKC-delta in chronically hypoxic rat myocardium: involvement in cardioprotection. Am J Physiol Heart Circ Physiol 288:H1566-H1572. doi:10.1152/ajpheart.00586.2004

16. Neckar J, Papousek F, Novakova O, Ost'adal B, Kolar F (2002) Cardioprotective effects of chronic hypoxia and ischaemic preconditioning are not additive. Basic Res Cardiol 97:161-167

17. Petrovski G, Das S, Juhasz B, Kertesz A, Tosaki A, Das DK (2011) Cardioprotection by endoplasmic reticulum stress-induced autophagy. Antioxid Redox Signal 24 [Epub ahead of print]

18. Przyklenk K, Bauer B, Ovize M, Kloner RA, Whittaker P (1993) Regional ischemic 'preconditioning' protects remote virgin 
myocardium from subsequent sustained coronary occlusion. Circulation 87:893-899

19. Przyklenk K, Zhao L, Kloner RA, Elliott GT (1996) Cardioprotection with ischemic preconditioning and MLA: role of adenosine-regulating enzymes? Am J Physiol 271:H1004-H1014

20. Riches K, Morley ME, Turner NA, O'Regan DJ, Ball SG, Peers C, Porter KE (2009) Chronic hypoxia inhibits MMP-2 activation and cellular invasion in human cardiac myofibroblasts. J Mol Cell Cardiol 47:391-399. doi:10.1016/j.yjmcc.2009.06.002

21. Tang XL, Rokosh G, Sanganalmath SK, Yuan F, Sato H, Mu J, Dai S, Li C, Chen N, Peng Y, Dawn B, Hunt G, Leri A, Kajstura J, Tiwari S, Shirk G, Anversa P, Bolli R (2010) Intracoronary administration of cardiac progenitor cells alleviates left ventricular dysfunction in rats with a 30-day-old infarction. Circulation 121:293-305. doi:10.1161/CIRCULATIONAHA.109.871905

22. Tang YL, Zhu W, Cheng M, Chen L, Zhang J, Sun T, Kishore R, Phillips MI, Losordo DW, Qin G (2009) Hypoxic preconditioning enhances the benefit of cardiac progenitor cell therapy for treatment of myocardial infarction by inducing CXCR4 expression. Circ Res 104:1209-1216. doi:10.1161/CIRCRESAHA.109.197723

23. van den Doel MA, Gho BC, Duval SY, Schoemaker RG, Duncker DJ, Verdouw PD (1998) Hypothermia extends the cardioprotection by ischaemic preconditioning to coronary artery occlusions of longer duration. Cardiovasc Res 37:76-81 pii: S00086363(97)00222-8

24. van Vlimmeren MA, Driessen-Mol A, van den Broek M, Bouten CV, Baaijens FP (2010) Controlling matrix formation and crosslinking by hypoxia in cardiovascular tissue engineering. J Appl Physiol 109:1483-1491. doi:10.1152/japplphysiol.00571.2010
25. Walker DM, Pasini E, Kucukoglu S, Marber MS, Iliodromitis E, Ferrari R, Yellon DM (1993) Heat stress limits infarct size in the isolated perfused rabbit heart. Cardiovasc Res 27:962-967

26. Whittaker P (1999) Laser-mediated reversal of cardiac expansion after myocardial infarction. Lasers Surg Med 25:198-206 pii: 10.1002/(SICI)1096-9101(1999)25:3<198:AID-LSM2>3.0.CO; 2-R

27. Whittaker P, Przyklenk K (1994) Reduction of infarct size in vivo with ischemic preconditioning: mathematical evidence for protection via non-ischemic tissue. Basic Res Cardiol 89:6-15

28. Xu WQ, Yu Z, Xie Y, Huang GQ, Shu XH, Zhu Y, Zhou ZN, Yang HT (2011) Therapeutic effect of intermittent hypobaric hypoxia on myocardial infarction in rats. Basic Res Cardiol doi: 10.1007/s00395-011-0159-y

29. Yamashita N, Hoshida S, Taniguchi N, Kuzuya T, Hori M (1998) Whole-body hyperthermia provides biphasic cardioprotection against ischemia/reperfusion injury in the rat. Circulation 98:1414-1421

30. Zacharowski K, Otto M, Hafner G, Chatterjee PK, Thiemermann C (1999) Endotoxin induces a second window of protection in the rat heart as determined by using p-nitro-blue tetrazolium staining, cardiac troponin $\mathrm{T}$ release, and histology. Arterioscler Thromb Vasc Biol 19:2276-2280

31. Zhao L, Elliott GT (1999) Pharmacologic enhancement of tolerance to ischemic cardiac stress using monophosphoryl lipid A. A comparison with antecedent ischemia. Ann N Y Acad Sci 874:222-235 